\title{
DESIGN OF A ROBUST MODEL REFERENCE ADAPTIVE VOLTAGE CONTROLLER FOR AN ELECTRODYNAMIC SHAKER
}

\author{
Leandro Della Flora and Hilton Abílio Gründling \\ Grupo de Eletrônica de Potência e Controle, UFSM. 97105-900 Santa Maria - RS - Brazil \\ ldella@mail.ufsm.br and ghilton@ctlab.ufsm.br
}

\begin{abstract}
This paper presents in detail the design of a high performance sinusoidal voltage controller for switching-mode $\mathrm{AC}$ power amplifiers intended to drive electrodynamic shakers. To obtain good reference tracking performance and stability over a wide frequency range and guarantee robustness under the effects of parameter variations and harmonic distortions, a model reference adaptive control scheme is employed. A very simplified model of the electrodynamic shaker is considered. The back electromotive force is dealt as unknown disturbance and the inductance of the armature is not modeled. The control strategy is digitally implemented and the design procedure requires only basic information about nominal electrical parameters of the shaker and the power amplifier. Fixed and swept frequency experimental tests performed up to $2 \mathrm{kHz}$ demonstrate the feasibility of this solution. The implementation of the voltage controller is also described.
\end{abstract}

Keywords - Discrete-Time Control, Pulse-Width Modulated Inverters, Robust Adaptive Control, Shakers.

\section{INTRODUCTION}

Mechanical vibration testing is widely used to study the effects of vibration and to evaluate physical properties of materials and structures in a variety of applications that range from circuit boards and aircraft to turbines and home appliances. Generally, the purpose is to simulate the dynamic environment where the test article will be stored or used in service and then to find out if it will survive to the rigors of the storage or service conditions [1].

The equipment normally needed to execute vibration tests includes an electrodynamic vibration machine (shaker), a power amplifier and an acceleration controlling and monitoring system (Fig. 1). The test article is attached to the table of the shaker and an accelerometer mounted on this table is used for feedback purposes in a vibration control system. The output command $r$ generated by the acceleration controller is then employed as the power supply reference signal.

The main requirements of amplifiers designed to drive electrodynamic shakers are to present good voltage or current control characteristic and low harmonic distortion over a wide dynamic and frequency range (typically, $10 \mathrm{~Hz}$ to 2 $\mathrm{kHz}$ ). Depending on whether the amplifier output current $i_{o}$ or voltage $v_{o}$ is proportional to the reference voltage $r$, the mode of operation is characterized: current (or transconductance) and voltage mode, respectively. In current mode, the mechanical resonance of the shaker suspension is in general very lightly damped, which makes the vibration control pro-

Artigo submetido em 09/08/2007. Revisão em 20/02/2008. Aceito por recomendação do editor F. L. M. Antunes. blem even more complicated and restricts this mode to very specific cases where closed-loop acceleration control is not required (such as in modal testing). In voltage mode, on the other hand, the suspension resonance is strongly damped as long as the impedance of the shaker is low. This important feature was determinant to consolidate the voltage mode in the majority of the vibration tests [8].

In terms of voltage control of switching-mode power amplifiers, most of the developed control techniques address the requirements of good reference tracking and low harmonic distortion for low frequency applications such as motor drives and uninterruptible power supplies [2]-[5]. In case of electrodynamic shakers, however, good performance is required over a very higher dynamic and frequency range, which imposes some unusual demands such as the ability of dealing during the same test with the back electromotive force produced in the shaker armature coil (which increases its impedance according to the mass of the test load) and the effects of the high frequency resonance of the amplifier output filter. In addition, due to the wide frequency power excitation, the armature electrical parameters are not constant, which significantly change the nature of the shaker impedance according to the controlled frequency.

The literature reports only a few solutions about the control of power amplifiers intended to drive electrodynamic shakers. In [6] and [7], an analog sinusoidal current control scheme has been presented. This solution is based on a high switching frequency and relatively low output power PWM inverter (50 kHz, $400 \mathrm{VA}$ ). Despite of the good control characteristic demonstrated experimentally until $2 \mathrm{kHz}$, this proposal has a very restricted application range as a consequence of the current mode operation.

A more general solution consisting of a robust model reference adaptive voltage controller has been presented in [9]. This technique comprises a reduced-order model of the electrodynamic shaker, where the back electromotive force is dealt as unknown disturbance and the inductance of the armature is not modeled. The control strategy is digitally implemented and experimental tests performed in a $3 \mathrm{kVA}, 12$ $\mathrm{kHz}$ PWM inverter have demonstrated the feasibility of this solution for controlling the shaker sinusoidal voltage, but only at frequencies up to $1 \mathrm{kHz}$.

Then, to further explore the applicability of [9], this paper presents some modifications that expand to $2 \mathrm{kHz}$ the frequency range of the voltage controller. Differently from [9], the control structure, the parameter adaptation law and the design of the robust model reference adaptive controller are presented in detail. Some implementation features along with fixed and swept frequency experimental tests are also provided. The development of the acceleration control system is not approached in this paper. 


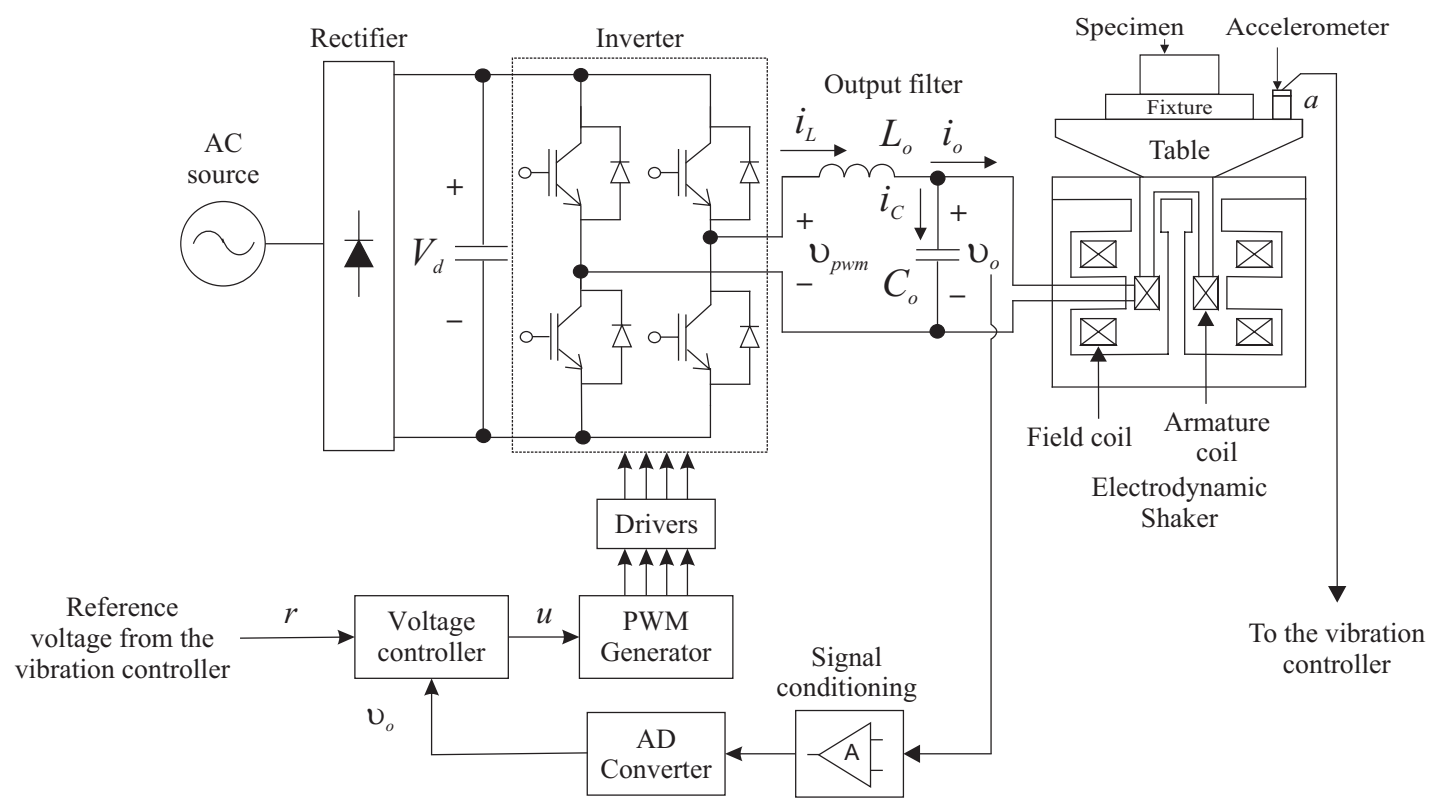

Fig. 1. The switching-mode AC power amplifier for excitation of an electrodynamic shaker.

\section{PLANT DESCRIPTION}

The schematic diagram of a switching-mode power amplifier designed to excite an electrodynamic shaker is shown in Fig. 1. It consists of a single-phase PWM inverter, a LC filter and a measuring and control platform. The inverter generates a pulse-width modulated voltage $v_{\text {pwm }}$ based on the control signal $u$. The output filter is used to reduce the harmonic content of the shaker input voltage $v_{o}$ in order to eliminate vibrations outside the main frequency range. The measuring and the control platform acquire the output voltage, compute the control law and generate the PWM signals.

The dynamic behavior of electrodynamic shakers is, in general, very complex due to resonances of the shaker body and moving mass. Unpredictable mechanical resonances and anti-resonances usually occur when elastic structures are attached to the table. The shaker electrical behavior, on the other hand, is determined not only by the moving coil resistance and inductance, but also by the back electromotive force generated across the coil. The mass of the device under test is variable and the coil resistance and inductance are not constant due to the wide frequency range excitation.

For voltage control purposes, however, the shaker impedance is not meaningfully affected by unknown mechanical resonances, which means that the modeling effort can be simplified, especially assuming that a robust control technique comprising reduced knowledge of the plant is to be developed. In this case, the following assumptions are stated:

(i) The radial magnetic flux density is constant.

(ii) The shaker body is rigidly attached to the floor.

(iii) Displacements between the shaker coil, table, fixture and test load are not modeled.

By doing so, the shaker moving element is dealt as a rigid mass and its dynamic behavior is approximately represented by the single degree-of-freedom system of indicated in Fig. 2(a). The mechanical and electrical equations that govern this system are:

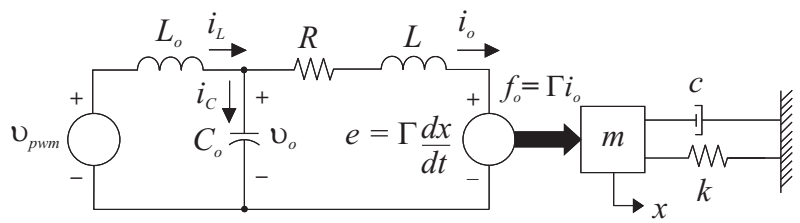

(a)

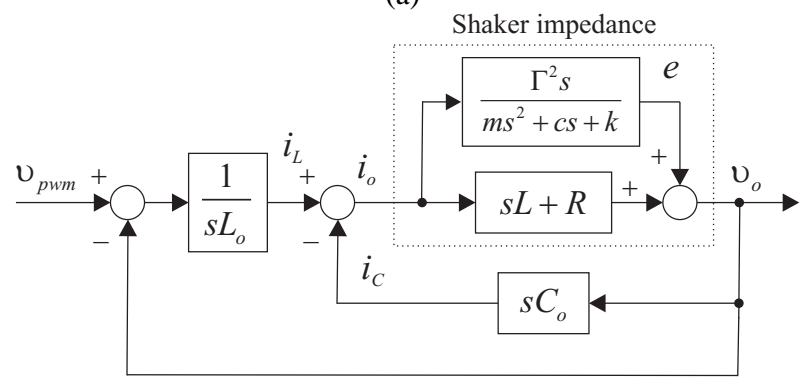

(b)

Fig. 2. A very simplified dynamic model: (a) Equivalent electromechanical circuit. (b) Transfer function block diagram.

$$
\begin{gathered}
v_{p w m}=L_{o} \frac{d i_{L}}{d t}+v_{o} \\
v_{o}=L \frac{d i_{o}}{d t}+R i_{o}+e \\
e=\Gamma \frac{d x}{d t} \\
C_{o} \frac{d v_{o}}{d t}=i_{C}=i_{L}-i_{o} \\
f_{o}=\Gamma i_{o}=m \frac{d^{2} x}{d t^{2}}+c \frac{d x}{d t}+k x
\end{gathered}
$$

Where:

$\Gamma \quad$ - force-generating constant $(\Gamma \triangleq B l)$.

$B \quad$ - magnetic flux density.

l - effective length of the armature conductors. 


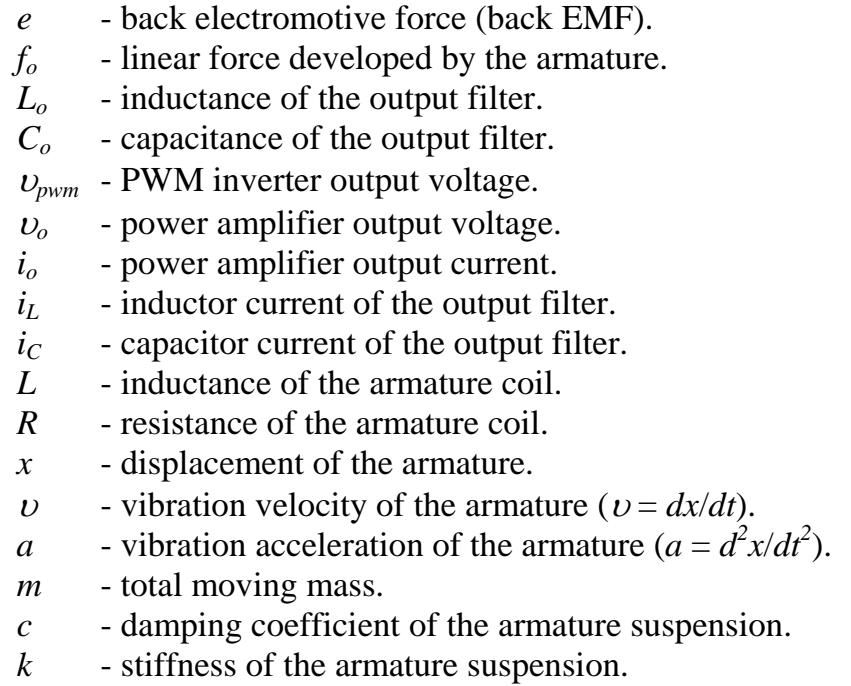

Despite most of these equations rely on basic electromechanical principles, special analysis is required for the back EMF. As the armature coil moves within the radial magnetic flux, a voltage is generated across the coil in proportion to the velocity of motion (3), which gives the active nature to the shaker. For a constant amplitude sinusoidal acceleration $a$, the motional EMF dominance in the equivalent circuit fades as the frequency increases, owing to the continual decrease in velocity $(v=a /(2 \pi f)$, where $f$ is the vibration frequency). Then, the armature coil reactance and resistance drops become significant and finally dominant. At very low frequencies $(0$ to $10 \mathrm{~Hz})$, the inductance drops are negligible and the equivalent circuit approximates to $R$ in series with the EMF generator $e$. At the suspension mass-spring resonance (typically $20 \mathrm{~Hz}$ ), motion can be sustained with virtually no current. Beyond this point, the EMF generator has a value inversely proportional to the vibration frequency. Above $1 \mathrm{kHz}$ or so, the equivalent circuit can then be approximated to one comprising $R$ and $L$ in series [11].

The back electromotive force reflects, in summary, the mechanical activity into the electrical circuit so that the shaker impedance changes from a predominantly capacitive load at low frequencies to a highly inductive nature over the high frequency range. Fig. 2(b) shows a linear transfer function block diagram of the corresponding switching-mode AC power amplifier when driving the electrodynamic shaker. The switching frequency is assumed to be much higher than the modulation frequency of the PWM inverter. Note that the moving mass $m$ influences $e$ and, since most sinusoidal vibration tests usually require constant magnitude acceleration with frequency variation at a specified rate in $\mathrm{Hz}$ or octave per minute, the amplitude of the back EMF and the shaker electrical parameters $R$ and $L$ are also time variant.

\section{ROBUST MODEL REFERENCE ADAPTIVE VOLTAGE CONTROLLER}

\section{A. Structure of the Controller}

Although electrodynamic shakers present a high degree of linearity between the input voltage $v_{o}$ and current $i_{o}$ if used within their specified force and motion limits, the operation over a wide frequency range combined to the meaningful variations on the shaker characteristics make it difficult to achieve good reference tracking and low harmonic distortion by using only fixed-gain controllers such as proportionalintegral-derivative (PID), PI, or P controller. An adaptive control scheme capable to automatically tune its gains and guarantee good performance and stability independently of the uncertainties presented, on the other hand, is recommended for such a case where the plant is strongly subjected to the effects of parameter variations. In this sense, the modeling effort can be further minimized by considering that:

(iv) The back EMF $e$ is an unknown disturbance.

(v) The inductance $L$ of the armature coil is not modeled.

Under these assumptions, the electrodynamic shaker is dealt as the resistive load $R$ shown in Fig. 3, i.e., the effect of $e$ into the output voltage $v_{o}$ is supposed to be mitigated by the robust adaptive controller and the effect of $v_{p w m}$ can be approximately represented as:

$$
G_{v}(s)=\frac{v_{o}(s)}{v_{p w m}(s)}=G_{v o}(s)\left[1+\mu \Delta_{m}(s)\right]
$$

where $G_{v}(s)$ is obtained by letting $e$ equal to zero in the block diagram of Fig. 2(b), i.e.:

$$
G_{v}(s)=\frac{s L+R}{s^{3} L_{o} C_{o} L+s^{2} L_{o} C_{o} R+s\left(L_{o}+L\right)+R}
$$

and the modeled part of the plant $G_{v o}(s)$ results by making $L$ equal to 0 in (7):

$$
G_{v o}(s)=\frac{R}{s^{2} L_{o} C_{o} R+s L_{o}+R}
$$

Then, $\mu \Delta_{m}(s)$ is a structured uncertainty on the model of the plant obtained by deliberately neglecting the inductance of the armature, i.e., $\mu=L$ is the neglected parameter and:

$$
\begin{gathered}
\mu \Delta_{m}(s)=\frac{G_{v}(s)-G_{v o}(s)}{G_{v o}(s)} \\
=\frac{s^{2} L_{o} \mu}{R\left[s^{3} L_{o} C_{o} \mu+s^{2} L_{o} C_{o} R+s\left(L_{o}+\mu\right)+R\right]}
\end{gathered}
$$

is the resulting multiplicative modeling error.

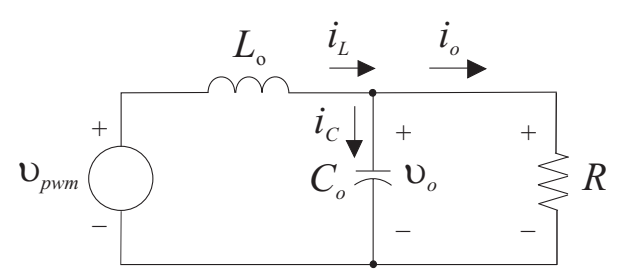

Fig. 3. The nominal load of the AC power amplifier.

The inverter power switches are turned on and off at each sampling interval $T$ so that $v_{p w m}$ is a voltage pulse of magnitude $-V_{d}$ or $V_{d}$ and width $\Delta T$. This analysis, however, assumes that the inverter modulator signal $u$ of Fig. 1 (control signal) is the average value of the voltage pulse in a sampling period. Thus, a discrete-time transfer function of (8) can be easily obtained by using a zero-order-hold with an appropriate sampling time [12]:

$$
G_{v o}(z)=\frac{v_{o}(z)}{u(z)}=k_{p} \frac{Z_{o}(z)}{R_{o}(z)}=k_{p} \frac{z+b_{1}}{z^{2}+a_{1} z+a_{2}}
$$

where $Z_{o}(z)$ and $R_{o}(z)$ are monic polynomials of degree $m_{p}$ and $n_{p}$, respectively. 
A model reference adaptive controller applicable to reduced-order plants and robust with respect to multiplicative stable modeling errors and bounded disturbances has been developed in [13] and [14]. This scheme, however, is only applicable to plants satisfying the following assumptions:

A1: $Z_{o}(z)$ is a monic Hurwitz polynomial of degree $m_{p} \leq$ $n_{p}-1$;

A2: $R_{o}(z)$ is a monic polynomial of degree $n_{p}$;

A3: the sign of $k_{p}$ and the values of $m_{p}$ and $n_{p}$ are known;

A4: $\Delta_{m}(z)$ is a stable transfer function;

A5: An upper bound $1>p_{o}>0$ on the stability margin $\mathrm{p}>$ 0 for which the poles of $\Delta_{m}(z / p)$ are stable is known.

For the modeled part of the plant, assumptions $A 1$ to $A 3$ are easily verified according to (10). Assumptions $A 4$ and $A 5$, on the other hand, are satisfied based on a discrete-time representation of $\mu \Delta_{m}(s)$.

The adaptive control objective consists, basically, in determining an appropriate control parameter vector $\boldsymbol{\theta}$ so that the resulting closed-loop plant is stable and the plant output $v_{o}$ tracks the reference model output $v_{m}$ as closely as possible, i.e., the desired closed-loop performance is clearly expressed in terms of the reference model choice:

$$
W_{m}(z)=\frac{v_{m}(z)}{r(z)}=k_{m} \frac{Z_{m}(z)}{R_{m}(z)}
$$

where $Z_{m}(z)$ and $R_{m}(z)$ are arbitrary monic Hurwitz polynomials of degrees $m_{p}$ and $n_{p}$, respectively, and $r$ is an uniformly bounded reference.

To achieve the adaptive control objective, the input $u$ and the output $v_{o}$ are used to generate the following auxiliary vectors:

$$
\begin{gathered}
\omega_{1}(k+1)=\mathbf{F}_{d} \omega_{1}(k)+\mathbf{q}_{d} u(k) \\
\omega_{2}(k+1)=\mathbf{F}_{d} \omega_{2}(k)+\mathbf{q}_{d} v_{o}(k)
\end{gathered}
$$

where $\mathbf{F}_{d}$ is a $\left(n_{p}-1\right) \times\left(n_{p}-1\right)$ stable matrix and $\left(\mathbf{F}_{d}, \mathbf{q}_{d}\right)$ is a controllable pair. Then, the input control law results as:

$$
u(k)=\boldsymbol{\theta}^{\mathrm{T}}(k) \boldsymbol{\omega}(k)+c_{o} r(k)
$$

where $\boldsymbol{\theta}^{\mathrm{T}}(k)=\left[\begin{array}{lll}\boldsymbol{\theta}_{1}^{\mathrm{T}}(k) & \boldsymbol{\theta}_{2}^{\mathrm{T}}(k) & \theta_{3}(k)\end{array}\right]$ is the $\left(2 n_{p}-1\right)$ control parameter vector, $\boldsymbol{\omega}^{\mathrm{T}}(k)=\left[\begin{array}{lll}\boldsymbol{\omega}_{1}^{\mathrm{T}}(k) & \boldsymbol{\omega}_{2}^{\mathrm{T}}(k) & v_{o}(k)\end{array}\right]$ and $c_{o}$ is a scalar feedforward parameter.

The augmented error $\varepsilon_{1}$ used for parameter adaptation can be stated as in [13]:

$$
\varepsilon_{1}(k)=v_{o}(k)-v_{m}(k)+\boldsymbol{\theta}^{\mathrm{T}}(k) \zeta(k)-v(k)
$$

where $\zeta=W_{m}(z) \mathbf{I} \boldsymbol{\omega}$ and $v=W_{m}(z) \boldsymbol{\theta}^{\mathrm{T}} \boldsymbol{\omega}$.

Fig. 4 shows a block diagram of the corresponding robust model reference adaptive voltage controller. Note that the control parameters are tuned based on the algorithm described next. Also, according to [15], the pair $\left(\mathbf{F}_{d}, \mathbf{q}_{d}\right)$ corresponds to a discrete-time approximation of $(\mathbf{F}, \mathbf{q})$, which in turn is a state space realization of $\alpha(s) / \Lambda(s)$, i.e.:

$$
(s \mathbf{I}-\mathbf{F})^{-1} \mathbf{q}=\frac{\alpha(s)}{\Lambda(s)}=\frac{\boldsymbol{\omega}_{1}}{u}=\frac{\boldsymbol{\omega}_{2}}{v_{o}}
$$

where $\Lambda(s)$ is an arbitrary monic Hurwitz polynomial of degree $n_{p}$ whose roots are commonly designed based on the desired closed-loop bandwidth.

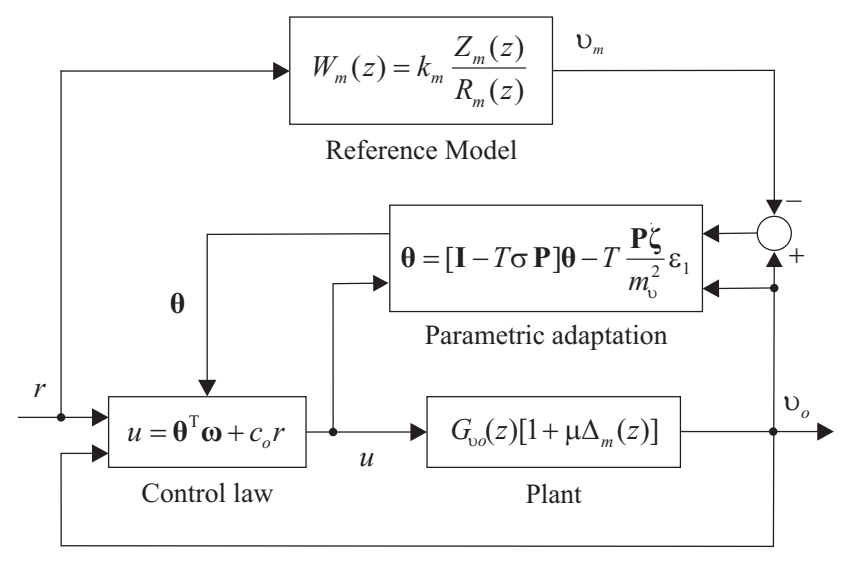

Fig. 4. The robust model reference adaptive controller.

\section{B. Parameter Adaptation Algorithm}

A modified least-squares algorithm subjected to assumptions $A 1$ to $A 5$ has been presented in [16]. The proposed solution exhibits fast parameter convergence and robustness even under the effects of time delays usually present in digital implemented control systems. In this case, a recursive form can be obtained by using the forward difference approximation:

$$
\begin{gathered}
\boldsymbol{\theta}(k+1)=[\mathbf{I}-T \sigma \mathbf{P}(k)] \boldsymbol{\theta}(k)-T \frac{\mathbf{P}(k) \zeta(k)}{m_{v}^{2}(k)} \varepsilon_{1}(k) \\
\mathbf{P}(k+1)=-T\left[\begin{array}{c}
\left.\frac{\mathbf{P}(k) \zeta(k) \zeta^{\mathrm{T}}(k) \mathbf{P}(k)}{m_{v}^{2}(k)}+\frac{\mathbf{P}^{2}(k)}{R_{v}^{2}} \bar{\mu}^{2}\right] \\
+\left(1+T \lambda \bar{\mu}^{2}\right) \mathbf{P}(k)
\end{array}\right.
\end{gathered}
$$

where $\mathbf{P}(k)=\mathbf{P}^{\mathrm{T}}(k)$ is such that:

$$
\begin{gathered}
0<\mathbf{P}(0) \leq \lambda R_{v}^{2} \mathbf{I}, \quad \mu^{2} \leq k_{\mu} \bar{\mu}^{2} \\
m_{v}(k+1)=\left(1-T \delta_{0}\right) m_{v}(k)+T \delta_{1}\left(|u(k)|+\left|v_{o}(k)\right|+1\right) \\
m_{v}(0)>\frac{\delta_{1}}{\delta_{0}}
\end{gathered}
$$

and $\lambda, \bar{\mu}, R_{v}, \delta_{0}, \delta_{1}$ are positive constants, $\delta_{1} \geq 1$ and $\delta_{0}<1$ satisfies:

$$
\delta_{0} \delta_{2}>\max \left[p_{0}, q_{0}\right]
$$

where $\delta_{2}$ is a positive constant and $1>q_{0}>0$ is such that the poles of $W_{m}\left(z / q_{0}\right)$ and $\left[\left(z / q_{0}\right) \mathbf{I}-\mathbf{F}_{d}\right]^{-1}$ are stable, $p_{0}$ is defined in $A 5$ and the $\sigma$-modification function is given by:

$$
\sigma=\left\{\begin{array}{lllc}
0 & \text { if } & \|\theta(k)\|<M_{0} \\
\sigma_{0} & \left(\frac{\|\theta(k)\|}{M_{0}}-1\right) & \text { if } & M_{0} \leq\|\theta(k)\| \leq 2 M_{0} \\
\sigma_{0} & \text { if } & \|\theta(k)\|>2 M_{0}
\end{array}\right.
$$

where $M_{0}$ is an upper bound for the norm of the desired control parameter vector $\boldsymbol{\theta}^{*}$ and $\sigma_{0}>2 \bar{\mu}^{2} / R_{v}^{2}$.

\section{Controller Design}

The design of the adaptive controller consists, mainly, in determining the desired closed-loop performance in terms of the reference model choice. Usually, there is no predefined 
rule for designing $W_{m}(z)$ but a common approach relates the reference model to the dynamic of the open loop system.

As an example, the electrodynamic shaker model St 5000/300 (manufactured by TIRA) and the power amplifier (Semikron, model SK45GB063) are considered. Fig. 5 shows the experimental variations of the shaker resistance $R$ and inductance $L$ produced as consequence of the skin effect. The design of the voltage controller, however, considers only the nominal parameters indicated in Table I (symbols with over line bar indicate nominal value).
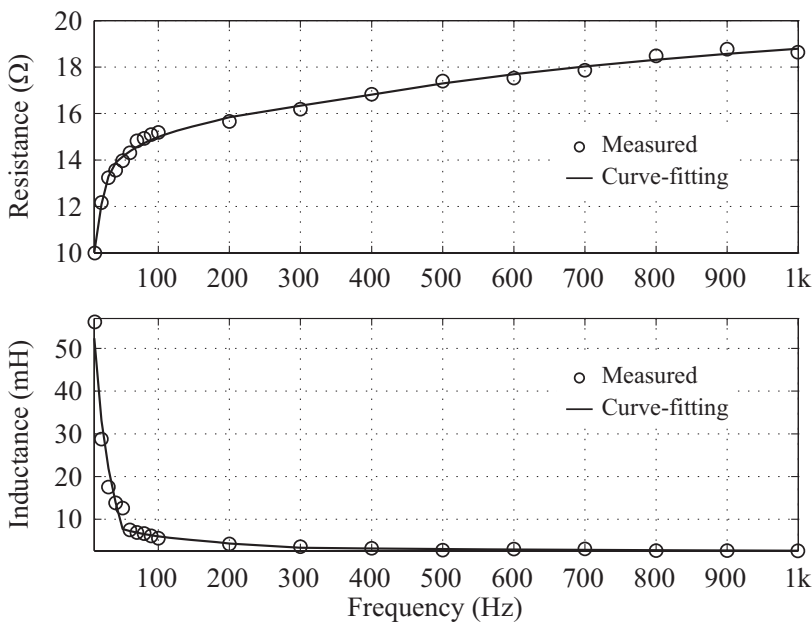

Fig. 5. The armature resistance and inductance.

TABLE I

Nominal electrical parameters

\begin{tabular}{ccc}
\hline $\bar{L}_{o}(\mu \mathrm{H})$ & $\bar{C}_{o}(\mu \mathrm{F})$ & $\bar{R}(\Omega)$ \\
\hline 250 & 10 & 12 \\
\hline
\end{tabular}

Based on these parameters, (8) becomes:

$$
G_{v o}(s)=\frac{4 \times 10^{8}}{s^{2}+8.33 \times 10^{3} s+4 \times 10^{8}}
$$

Then, selecting $W_{m}(s)$ with smaller rise and settling time than $G_{v o}(s)$ usually results in good closed-loop performance and avoids overloading the actuator during transient periods:

$$
W_{m}(s)=\frac{9.87 \times 10^{8}}{s^{2}+3.96 \times 10^{4} s+9.87 \times 10^{8}}
$$

A comparison between the step responses of $G_{v o}(s)$ and $W_{m}(s)$ in Fig. 6 indicates that the performance of the closedloop system is now mainly dependent on how the nominal model of the plant represents the actual electrodynamic shaker and, more importantly, on how the parameter adaptation algorithm can deal with differences.

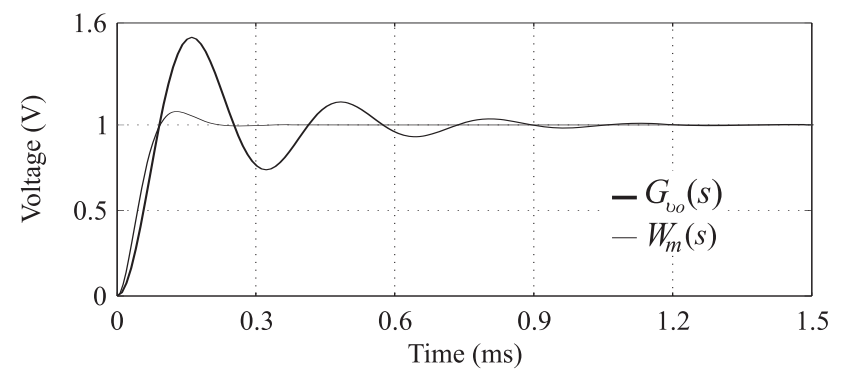

Fig. 6. Step responses of $G_{v o}(s)$ and $W_{m}(s)$.
The digital implementation of the adaptive controller is straightly linked to the sampling frequency choice. A good rule for setting the sampling time $T$ consists in determining the bandwidth of the desired closed-loop system and then to make $1 / T$ about 6 to 25 times greater than the corresponding bandwidth [12]. Obviously, the sampling rate influences properties like the command signal tracking, the rejection of disturbances and measurement noise, the sensitivity to unmodeled dynamics and the computer power required.

In the current case, the system is designed to operate according to the low frequency limit of the electrodynamic shaker $(20 \mathrm{~Hz})$ and the upper limit of the power amplifier (2 $\mathrm{kHz}$ ). Then, for ease of implementation, the sampling frequency $(1 / T)$ is chosen equal to the inverter switching rate $(24 \mathrm{kHz})$ so that a balance between the closed-loop bandwidth criteria and the switching frequency is achieved.

The discrete-time equivalent representation of (22) and (23) is obtained by applying z-transformation to the zeroorder-hold/transfer function product, i.e.:

$$
\begin{gathered}
G_{v o}(z)=0.293 \frac{z+0.888}{z^{2}-1.153 z+0.707} \\
W_{m}(z)=0.467 \frac{z+0.565}{z^{2}-0.4614 z+0.1922}
\end{gathered}
$$

For a second order plant, the filter $\alpha(s) / \Lambda(s)$ of $u$ and $v_{o}$ in (15) is defined as in [15]:

$$
\frac{\alpha(s)}{\Lambda(s)}=\frac{q}{s-F}
$$

and the corresponding digital implementation $\left(F_{d}, q_{d}\right)$ is determined by applying a zero-order-hold to $(s I-F)^{-1} q$ :

$$
\begin{gathered}
F_{d}=e^{F T} \\
q_{d}=\left(e^{F T}-1\right) \frac{q}{F}
\end{gathered}
$$

To present a unitary gain at low frequencies and to avoid high frequency noise amplification in the control law, $\alpha(s) / \Lambda(s)$ is designed with $q$ set equal to $-F$ and the cutoff frequency $-F$ is defined as $2.000 \mathrm{rad} / \mathrm{s}$. Then, $F_{d}$ and $q_{d}$ result from (27) as 0.92 and 0.08 , respectively.

The modeled part $G_{v o}(s)$ of the plant has a magnitude increase at high frequency operation due to the resonance that occurs between the output filter inductor and capacitor. In practice, this amplification can be dealt as an increase of $k_{p}$ and necessity of reducing the control parameter $c_{o}$ as long as $k_{m}$ remains constant (according to the model reference control theory [15], $\left.c_{o}=k_{m} / k_{p}\right)$. Then, differently from [9]:

$$
c_{o}(f)=\left\{\begin{array}{ccc}
2.2-1.7^{f / 500} & \text { if } & f \leq 500 \\
0.5 & \text { if } & f>500
\end{array}\right.
$$

where $f$ is the frequency of the reference $r$.

For the parameter adaptation algorithm, the upper limits on the stability margin $p_{0}$ and $q_{0}$ need to be determined firstly. In case of $q_{0}$, considering the characteristic equations of $W_{m}(z)$ and $\alpha(z) / \Lambda(z)$ and substituting $z$ by $q_{0} z$, i.e.:

$$
\begin{gathered}
q_{0}^{2} z^{2}-0.4614 q_{0} z+0.1922=0 \\
q_{0} z-F_{d}=0
\end{gathered}
$$

the upper limit on the stability margin corresponds to the smallest value of $q_{0}$ between 0 and 1 so that the roots $z_{1}$ and 
$z_{2}$ of (29) and the root $z_{3}$ of (30) satisfy $\left|z_{i}\right|<1$ ( $i=1,2$ and

3). Then, by making $q_{0}$ to vary from 1 to 0 :

$$
q_{0}=0.92
$$

and both solutions of (29) and (30) lie within the unit circle of the $z$-plane.

The upper limit on the stability margin $p_{0}$ of $\mu \Delta_{m}(z)$, on the other hand, is related to the magnitude of the neglected parameter. Although the inductance of the armature is frequency dependent, the stability analysis is more representative by considering only the maximum value of $L$, which is measured at the lower frequency limit, i.e., $\bar{L}=55 \mathrm{mH}$ for model St 5000/300 in Fig. 5. Thus, from (9) and Table I:

$$
\mu \Delta_{m}(s)=\frac{8.33 \times 10^{3} s^{2}}{s^{3}+218.2 s^{2}+4.02 \times 10^{8} s+8.73 \times 10^{10}}
$$

or, equivalently:

$$
\mu \Delta_{m}(z)=0.3068 \frac{z^{2}-2 z+1}{z^{3}-2.333 z^{2}+2.33 z-0.991}
$$

and the upper bound on the stability margin $p_{0}$ is obtained similarly to $q_{0}$, but from the characteristic equation of $\mu \Delta_{m}(z)$ :

$$
p_{0}=0.99
$$

Based on (20), $\delta_{0}$ is defined equal to 0.991 and, since $\bar{\mu}$ is an upper bound for $L, \bar{\mu}$ is set equal to $100 \mathrm{mH}$. Due to the absence of a deterministic design procedure, other positive constants were determined arbitrarily according to the restrictions stated in (16) to (21) and evaluated by computer simulation under different conditions until good convergence rate was obtained. As an example, the initial value of the control parameter vector $\boldsymbol{\theta}(0)$ was firstly set equal to $\mathbf{0}$. Then, after some iterations, it was verified that good convergence is achieved by making $\boldsymbol{\theta}(0)$ equal to $\left[\begin{array}{lll}-1 & 0.3 & 0.7\end{array}\right]^{\mathrm{T}}$. In case of the desired control parameter vector $\boldsymbol{\theta}^{*}$, it is assumed that an upper bound $M_{0}$ for $\left\|\boldsymbol{\theta}^{*}\right\|$ is known. Nevertheless, it is shown in [13] that choosing $M_{0}$ as large as possible but smaller than $1 / \bar{\mu}$ always satisfies $M_{0}>\left\|\boldsymbol{\theta}^{*}\right\|$ in presence of unmodeled dynamics, provided that $\bar{\mu}$ is small. Table II

\begin{tabular}{|c|c|}
\hline Symbol & Value \\
\hline$\theta(0)$ & {$\left[\begin{array}{lll}-1 & 0.3 & 0.7\end{array}\right]^{\mathrm{T}}$} \\
\hline $\mathbf{P}(0)$ & $100 \times \mathbf{I}_{3 \times 3}$ \\
\hline$\zeta(0)$ & {$\left[\begin{array}{lll}0 & 0 & 0\end{array}\right]^{\mathrm{T}}$} \\
\hline $\mathbf{F}_{d}$ & 0.92 \\
\hline $\mathbf{q}_{d}$ & 0.08 \\
\hline$\omega_{1}(0)$ & 0 \\
\hline$\omega_{2}(0)$ & 0 \\
\hline$\lambda$ & 10 \\
\hline $\bar{\mu}$ & 0.1 \\
\hline$R_{v}$ & 10 \\
\hline$\delta_{0}$ & 0.991 \\
\hline$\delta_{1}$ & 1 \\
\hline$M_{0}$ & 9 \\
\hline$\sigma_{0}$ & 0.1 \\
\hline$T$ & $41.66 \mu \mathrm{s}$ \\
\hline
\end{tabular}
presents the designed controller parameters.

TABLE II

Designed controller parameters

\section{IMPLEMENTATION AND EXPERIMENTAL RESULTS}

\section{A. The System Configuration and Digital Implementation}

The electrodynamic shaker considered in the controller design is a $1 \mathrm{kVA}$ vibration machine capable to operate from $20 \mathrm{~Hz}$ to $5 \mathrm{kHz}$. The maximum sine force peak is approximately $3 \mathrm{kN}$, the mass of the armature and fixture assembly is equal to $22 \mathrm{~kg}$ and the maximum specimen weight is 46 $\mathrm{kg}$. The power amplifier, on the other hand, generates sinusoidal voltages between $10 \mathrm{~Hz}$ and $2 \mathrm{kHz}$ with a maximum peak of the output voltage $v_{o}$ equal to $110 \mathrm{~V}$. Then, based on the vibration machine and the power supply constraints, the frequency range was set equal to $20 \mathrm{~Hz}$ to $2 \mathrm{kHz}$ and the maximum voltage and current amplitudes were defined as $110 \mathrm{~V}$ and $20 \mathrm{~A}$, respectively.

A multifunction interface board was used to communicate the PC-based control platform and the AC power amplifier. The control routine was developed in Borland C programming language to acquire the external variables and to compute the control law according to the specified voltage amplitude and frequency. The control signal $u$ was converted into a PWM signal and then applied to the AC power amplifier module through the multifunction interface board (Fig. 1). The robust adaptive algorithm was implemented as:

1) Calculate $r(k)$ and $v_{m}(k)$;

2) Acquire the external variable $v_{o}(k)$;

3) Compute the control law $u(k)$;

4) Generate the PWM signals;

5) Calculate $\omega_{1}(k+1), \omega_{2}(k+1), \varepsilon_{1}(k+1), \sigma, \boldsymbol{\theta}(k+1)$, $\mathbf{P}(k+1), \zeta(k+1), v(k+1)$ and $m_{v}(k+1)$.

\section{B. Experimental Results}

The experimental performance of the robust adaptive voltage controller has been evaluated first with a $24 \Omega$ resistive load. Figs. 7(a) and 7(b) show the corresponding power amplifier output voltage $v_{o}$ and the reference model $v_{m}$ at $20 \mathrm{~Hz}$ and $2 \mathrm{kHz}$, respectively. Then, the control scheme has been applied to compensate the tracking error between $v_{o}$ and $v_{m}$ when the electrodynamic shaker is inserted (see Fig. 8). Fig. 9 shows results at different amplitudes. Note that due to the limited number of points acquired per cycle in high frequencies, the voltage waveform appears to be distorted in $2 \mathrm{kHz}$, which is absolutely not verified in the analog domain.

To verify the transient tracking response with frequency varying continuously, a constant amplitude voltage has been employed to drive the electrodynamic shaker. The resonant search test requirements specified in [17] have been taken as a practical example of frequency sweep. According to these specifications, the device under test must be submitted to a sinusoidal logarithmic frequency sweep at the rate of 1 octave per minute, which demands approximately 7 minutes to complete a whole sweep between $20 \mathrm{~Hz}$ and $2 \mathrm{kHz}$. Then, to facilitate the exposition of the results obtained, some particular frequencies were selected and combined in the same figure. Figs. 10(a) and 10(b) show the corresponding measured voltage waveforms from $30 \mathrm{~Hz}$ to $100 \mathrm{~Hz}$ and from $600 \mathrm{~Hz}$ to $2 \mathrm{kHz}$, respectively. 


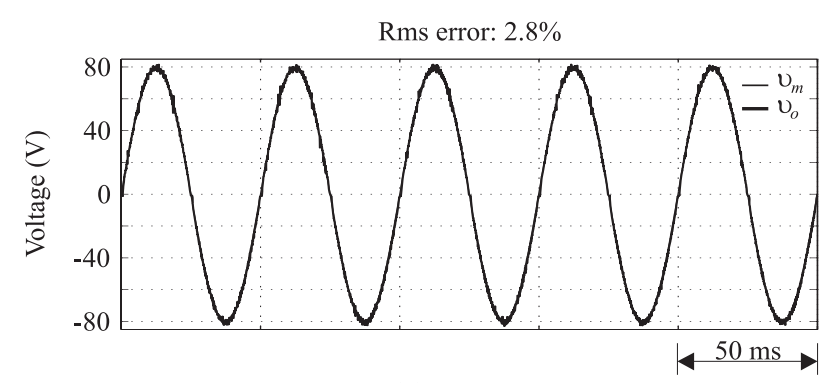

(a)

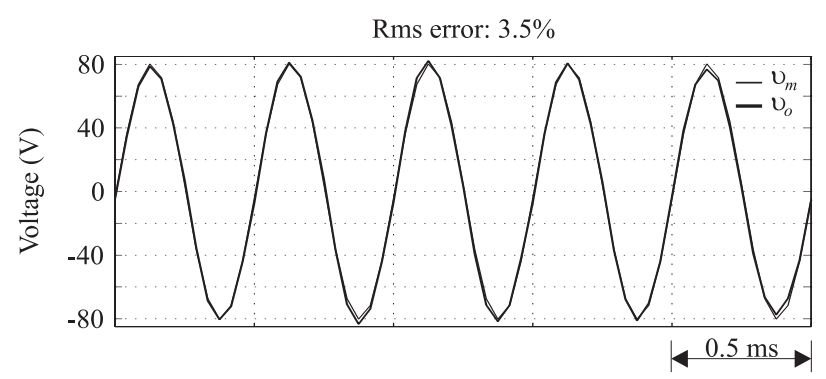

(b)

Fig. 7 Reference model output $v_{m}$ and the measured voltage waveform $v_{o}$ with a $24 \Omega$ resistive load. (a) $20 \mathrm{~Hz}$. (b) $2 \mathrm{kHz}$.

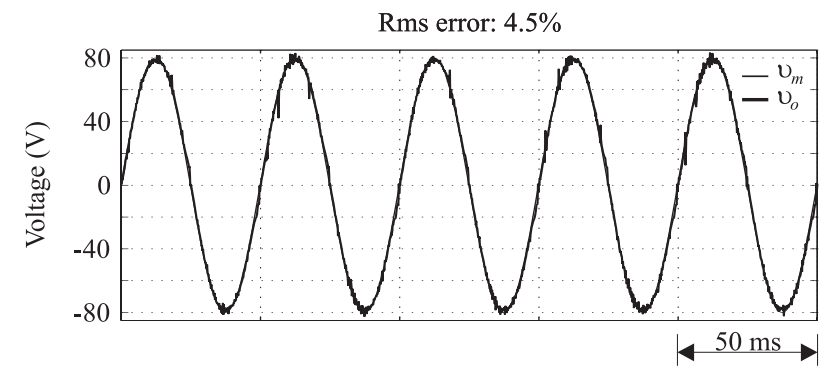

(a)

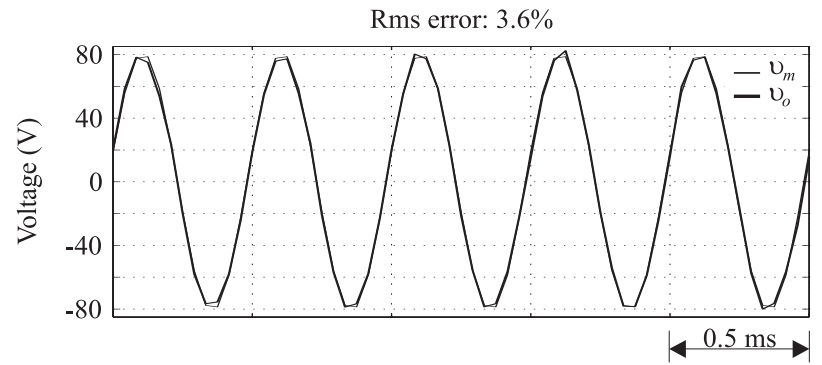

(b)

Fig. 8 Reference model output $v_{m}$ and the measured voltage waveform $v_{o}$ with the electrodynamic shaker. (a) $20 \mathrm{~Hz}$. (b) $2 \mathrm{kHz}$.

By taking into account the meaningful changes that occur on the shaker dynamic behavior as function of the excitation frequency, the experimental results demonstrated the feasibility of the this solution in controlling the armature voltage both at low and high frequencies and with fixed or swept frequency tests. In addition, it is important to note that, due to the reduced order model considered, the design of the robust adaptive controller requires only basic information about nominal electrical parameters of the shaker and the power amplifier.

\section{CONCLUSION}

The development of a high performance voltage controller for switching-mode AC power amplifiers intended to drive electrodynamic shakers is a very important issue. Low harmonic distortion and good reference tracking are required over a wide dynamic and frequency range. In addition, the system is strongly subjected to the effects of the back electromotive force and the parameters variations.

This paper has presented a sinusoidal voltage controller based on a robust model reference adaptive technique comprising reduced-order model of the shaker. The back electromotive force is dealt as unknown disturbance and the inductance of the armature is not modeled. The controller is digitally implemented and the design requires only basic information about nominal electrical parameters of the shaker and the power amplifier. Fixed and swept frequency experimental tests executed up to $2 \mathrm{kHz}$ have demonstrated the high performance of this controller in adjusting the voltage of the shaker armature. Topics for future research include the development of an acceleration control scheme whose output command corresponds to the reference signal of the power amplifier.

\section{ACKNOWLEDGEMENT}

This work was supported by CNPq (Conselho Nacional de Desenvolvimento Científico e Tecnológico), UFSM (Universidade Federal de Santa Maria) and GEPOC (Grupo de Eletrônica de Potência e Controle).

\section{REFERENCES}

[1] C. M. Harris, Shock and Vibration Handbook, McGrawHill, 5th ed, New York, 2001.

[2] Y.-Y. Tzou, R.-S. Ou, S.-L. Jung, M.-Y. Chang, "HighPerformance Programmable AC Power Source with Low Harmonic Distortion Using DSP-Based Repetitive Control Technique", IEEE Transactions on Power Electronics, vol. 12, no. 4, pp. 715-725, April 1997.

[3] K. S. Low, "A DSP-Based Single-Phase AC Power Source," IEEE Transactions on Industrial Electronics, vol. 46, no. 5, pp. 936-941, May 1999.

[4] E. G. Carati, H. Pinheiro, J. R. Pinheiro, H. L. Hey, H. A. Gründling, "Adaptive Robust DSP-Based Single Phase AC Power Source", in Proc. of International Conference on Control Applications, pp. 24-28, 2001.

[5] C. Rech, H. Pinheiro, H. A. Gründling, H. L. Hey, J. R. Pinheiro, "A Modified Discrete Control Law for UPS Applications”, IEEE Transactions on Power Electronics, vol. 18, no. 5, pp. 1138-1145, May 2003.

[6] T. H. Chen, K. C. Huang, C. M. Liaw, "A HighFrequency Switching-Mode Power Amplifier for Shaker Armature Excitation”, IEE Electric Power Applications, vol. 144, no. 6, pp. 415-422, November 1997.

[7] T. H. Chen, C. M. Liaw, "Vibration Acceleration Control of an Inverter-Fed Electrodynamic Shaker", 


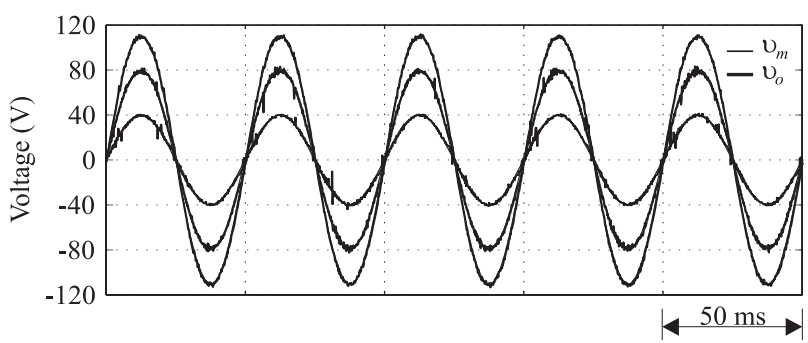

(a)

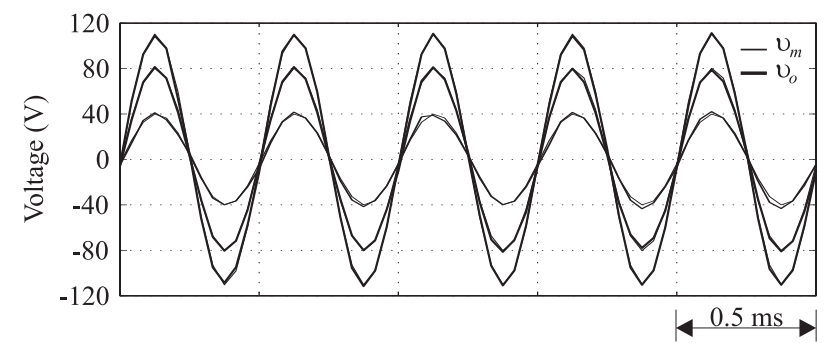

(b)

Fig. 9 Reference model output $v_{m}$ and the measured voltage waveform $v_{o}$ with different amplitudes. (a) $20 \mathrm{~Hz}$. (b) $2 \mathrm{kHz}$.

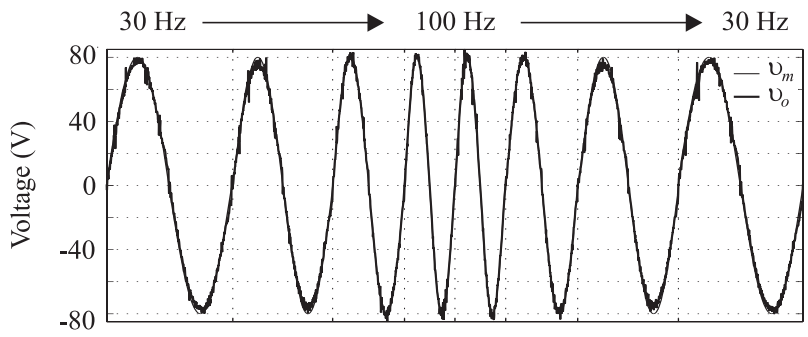

(a)

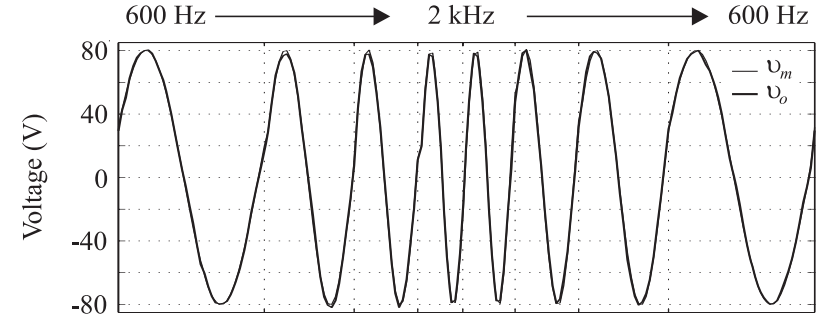

(b)

Fig. 10 Reference model output $v_{m}$ and the measured voltage waveform $v_{o}$ with swept frequency. (a) 30 to $100 \mathrm{~Hz}$. (b) $600 \mathrm{~Hz}$ to $2 \mathrm{kHz}$.

IEEE/ASME Transactions On Mechatronics, vol. 4, no. 1, pp. 60-70, March 1999.

[8] W. Tustin, Random Vibration \& Shock Testing, Equipment Reliability Institute, 1st ed, Santa Barbara, 2005.

[9] L. Della Flora, H. A. Gründling, "Digital Environment for Sinusoidal Vibration Test Control of an AC Power Source-Fed Electrodynamic Shaker", Revista Eletrônica de Potência, vol. 11, no.3, pp. 167-174, November 2006.

[10] —, "Vibration Acceleration Control of an AC Power Source-Fed Electrodynamic Shaker", in Proc. of Power Electronics Specialists Conference, vol. 37, pp. 11751181, 2006.

[11] R. Fair, H. R. Bolton, “Analysis and Design of Electromagnetic Moving Coil Vibration Generators", in Proc. of International Electrical Machines and Drives Conference, vol. 6, pp. 529-534, 1993.

[12] K. J. Astrom, B. Wittenmark, Computer-Controlled Systems, Prentice Hall, 3rd ed. Upper Saddle River, 1997.

[13] P. Ioannou, K. Tsakalis, “A Robust Direct Adaptive Controller", IEEE Transactions on Automatic Control, vol. 31, no. 11, pp. 1033-1043, November, 1986.

[14] _ - "A Robust Discrete-Time Adaptive Controller”, in Proc. of Conference on Decision and Control. Athens, vol. 29, pp. 838-843, 1986.

[15] P. A. Ioannou, J. Sun, Robust Adaptive Control, Prentice Hall, 1st ed. Upper Saddle River, 1996.

[16] R. Lozano-Leal, J. Collado, S. Mondié, "Model Reference Adaptive Control Without a Priori Knowledge of the High Frequency Gain”, IEEE Transactions on Automatic Control, vol. 35, no. 1, pp. 71-78, January 1990.

[17] Environmental Considerations in Development of Mobile Agricultural Electrical/Electronic Components, ANSI/ASAE EP455, St Joseph, July 1991.

\section{BIOGRAPHIES}

Leandro Della Flora was born in São Borja, RS, Brazil, in 1979. He received the B.S. and M.S. degree in electrical engineering from the Universidade Federal de Santa Maria, Santa Maria, Brazil, in 2003 and 2005, respectively.

He is currently working towards his Ph.D. degree at the same institution. His areas of research interest include control systems, power electronics and instrumentation applied to vibration testing using electrodynamic shakers.

M. Eng. Della Flora is an active student member of the Brazilian Power Electronics Society.

Hilton Abílio Gründling was born in Santa Maria, RS, Brazil, in 1954. He received the B.S. degree from the Pontifícia Universidade Católica do Rio Grande do Sul, Porto Alegre, Brazil, in 1977, the M.S. degree from the Universidade Federal de Santa Catarina, Florianópolis, Brazil, in 1980 and the Ph.D. degree from the Instituto Tecnológico de Aeronáutica, São Paulo, Brazil, in 1995.

Since 1980, he has been with the Universidade Federal de Santa Maria, Santa Maria, Brazil, where he is currently a Professor. His research interests include robust model reference adaptive control, discrete-time control and control systems applications. 